\title{
The reduction of peak overpressure using concrete blast barriers
}

\author{
R. Hájek \& M. Foglar \\ Department of Concrete and Masonry Structures, \\ Faculty of Civil Engineering, Czech Technical University in Prague, \\ Czech Republic
}

\begin{abstract}
Due to the rise of terroristic attack threat, public buildings should be designed to ensure as much safety of its visitors as possible. This paper is focused mainly on understanding of blast wave dynamics in interaction with the solid building and its equipment. Especially pressure wave reflections inside a confined space are of particular interest. The effect of atmospheric overpressure on the human body is also studied. A method for computer modelling of explosive events was developed using LS-DYNA software. The FEM model was calibrated based on experimental data. Multiple arrangements and shapes of concrete barriers were then proposed for faster energy dissipation and effective reduction of the damage and injuries caused by the explosion. The most promising arrangements according to FEM modelling results were then selected for the experimental program. This paper also includes a comparison of the experimental data and results of numerical FEM analysis. The accuracy of the FEM model is then discussed. Thanks to use of TNT explosive charge, the experimental results can also be compared to other blast experiments without barriers. The effectiveness of different barrier arrangements are compared and evaluated.

Keywords: explosion, air blast wave, overpressure, barrier.
\end{abstract}

\section{Introduction}

Due to rise of the threat of terrorist attack the research in the field of blast loading on structures and their interiors has received considerable attention in recent years. Buildings such as railway stations, airports or embassies ought to be designed to ensure as much safety of occupants as possible. Structure shall 
not collapse due to any of considerable situations. Furthermore, measures shall be taken to reduce severity of the explosion (magnitude of the pressure wave coming from epicentre of the explosion and the amount of potentially harmful flying debris).

Zhou and Hao [1] already studied blast loading on buildings and possibilities of protection of buildings against explosion. It was proved that a barrier can affect propagation of the blast wave. This research is focused on reduction of peak overpressure at the front of the passing blast wave by a system of blast barriers situated in the large hall of a public building such as railway station. Reduction of the overpressure leads to reduction of severity of injuries to occupants of the building. For the purpose of research the shape of pressure disturbance at any location away from the blast was simplified as seen in Figure 1. Table 1 shows the approximate values of peak overpressure that result in injury to people.

Table 1: Overpressure effects on human body [2].

\begin{tabular}{|c|c|}
\hline Peak overpressure $\Delta \mathbf{p}[\mathbf{k P a}]$ & Impact on the human body \\
\hline 16.5 & Eardrums damage at $1 \%$ affected \\
\hline 19.3 & Eardrums damage at $10 \%$ affected \\
\hline 34.5 & Eardrums damage at $50 \%$ affected \\
\hline 43.4 & Lungs damage threshold \\
\hline 100.0 & Fatal injuries at $1 \%$ affected \\
\hline 120.7 & Fatal injuries at $10 \%$ affected \\
\hline 141.3 & Fatal injuries at $50 \%$ affected \\
\hline 175.8 & Fatal injuries at $90 \%$ affected \\
\hline 200.0 & Fatal injuries at $99 \%$ affected \\
\hline
\end{tabular}

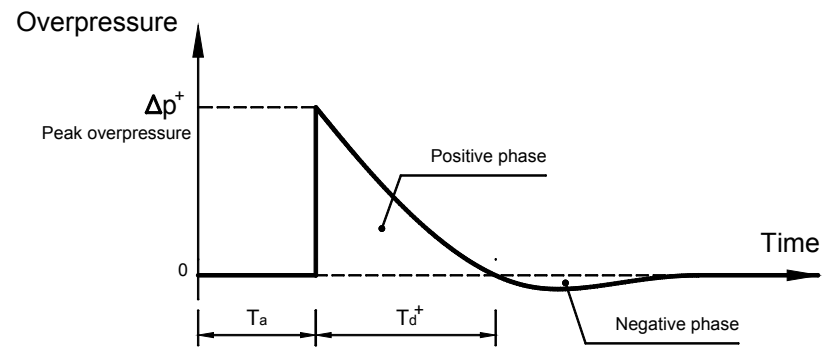

Figure 1: Idealized shape of the air shock wave.

Arrangement of blast barriers between the explosive and a victim has great impact on effectiveness of the barriers. Barriers should be arranged as effectively as possible. On the other hand, due to the nature of terrorist attack it is impossible to predict position of the explosive within the building so the arrangement of barriers has to be universal. 
The main reason of barriers is to divide the open space into smaller segments. When the explosion occurs, surface areas in which the overpressure reaches harmful levels are decreased. It means that the possibility of injury would be reduced. To study the effect of barriers on overpressure reduction, an example was proposed where explosive device is planted in the most populated area, presumably in front of information boards.

The research is based on computer modelling of explosive event using finite element method, more precisely the LS-DYNA solver. Modelling is backed up by comparison to experimental data [3,4].

An original experimental programme was proposed in order to determine ways to influence propagation of the blast wave by a system of concrete blast barriers and to validate results obtained from computer analysis.

\section{Experimental program}

Proposed experiments were performed by the Institute of Energetic Materials, Faculty of Chemical Technology, University of Pardubice, Czech Republic. Prior to the experiment itself a numeric study was conducted to predict behaviour of pressure wave in interaction with solid barrier and multiple arrangements of blast barriers were designed. Pure TNT charge was used as an explosive. That means the obtained results can be easily compared to experimental data from other authors. Pachman et al. [4] conducted a series of experiments with varying yield and standoff distance from the explosive charge.

\subsection{Scaling}

Given the typical dimensions of public buildings and the predicted maximal weight of the explosive charge to be carried by a single person it was decided to conduct the experiments in reduced scale $1: n$. Widely used scaling laws $[5,6]$ state:

$$
\begin{aligned}
A_{\text {real }} & =A_{\text {model }} \cdot n \\
W_{\text {real }} & =W_{\text {model }} \cdot n^{3}
\end{aligned}
$$

where $n$ is a scaling factor, $A$ is a parameter of length and $W$ is weight of the explosive.

The scale used for experiment was determined to be 1:4. That means the weight of the explosive is reduced from $25 \mathrm{~kg}$ at the full scale to only $391 \mathrm{~g}$ at the reduced scale.

\subsection{Barrier arrangement}

Multiple arrangements of barriers were evaluated numerically using FEM analysis software LS-DYNA prior to the experiment. Some of the arrangements were chosen for the reduced scale experiments (Figure 2). Thanks to reduced scale of the experiment easily obtainable RC precast walls were used to model solid barriers (Figure 3). This solution was cheaper than custom made concrete panels. Use of precast walls also allowed for easier handling and repositioning. 


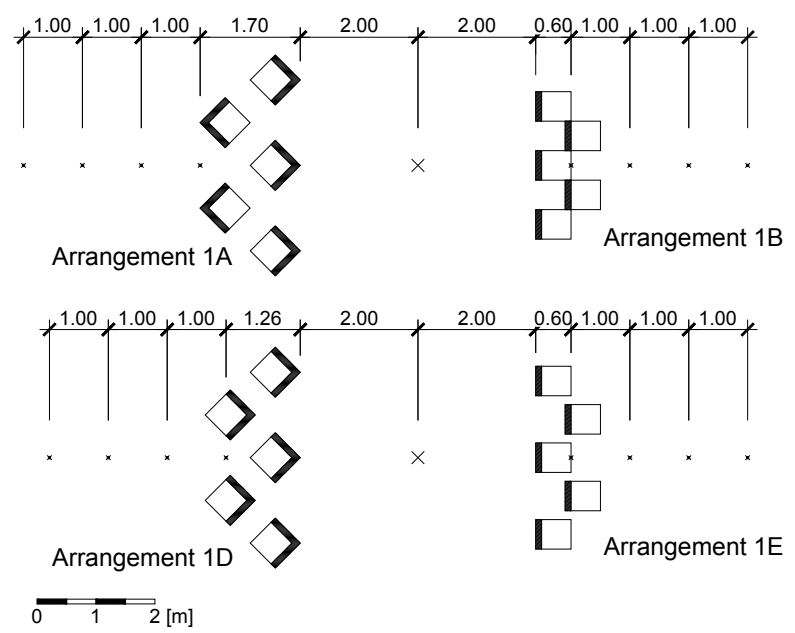

Figure 2: Tested arrangements of concrete barriers. Dimensions in metres.

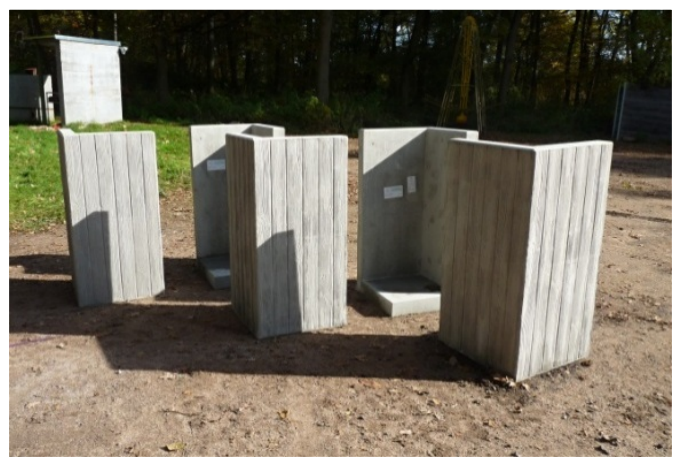

Figure 3: $\quad$ Barrier layout 1A set up on site.

One additional arrangement was considered (1C). Five precast walls were positioned in line and were equipped with custom made steel console at the top pointing at 45 degree angle towards the explosive. This arrangement was added to the original set because the computer modelling study predicted greater effectiveness of angled barrier shape than the basic rectangular shape used in arrangements $1 \mathrm{~A}, 1 \mathrm{~B}, 1 \mathrm{D}$ and $1 \mathrm{E}$.

\subsection{Data collection and results}

During the experiment pressure sensors were used to measure the peak overpressure at the front of passing air shock wave. For each tested arrangement four sensors were placed in various distances from the barriers as shown in Figure 1. Example of sensor output from one blast is shown in Figure 4. Each experiment layout was tested twice. 


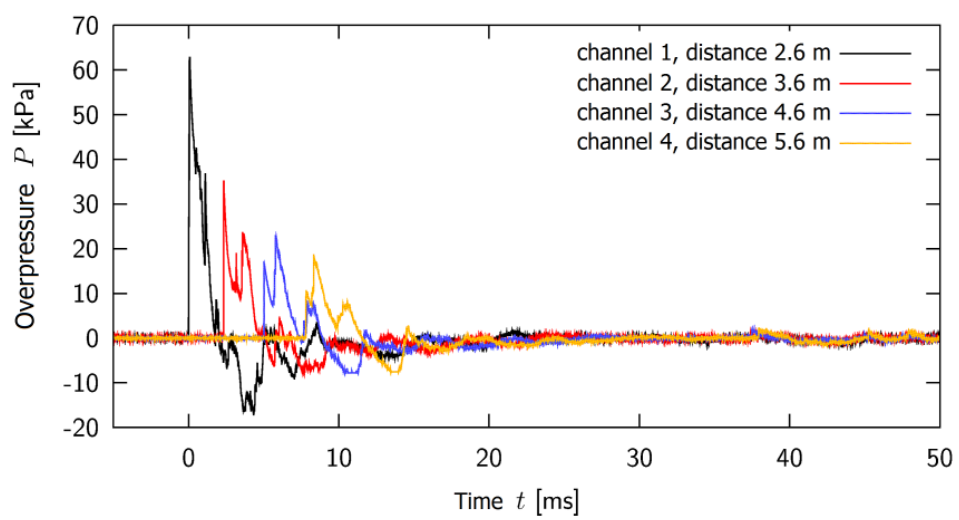

Figure 4: Example of overpressure measured during single blast in multiple standoff distances - arrangement 1E (308 g TNT).

The obtained results are summarized in Figure 5 and Figure 6. Both figures show comparison of measured peak overpressure at the front of passing air shock wave for each tested arrangement and reference arrangement without any barrier derived from [4]. Rectangular barriers (Figure 5) do not appear to have any significant effect on the value of peak overpressure. On the other hand, the angled barrier (Figure 6) has proven that there is a possibility of influencing significantly propagation of the pressure wave with rigid barriers.

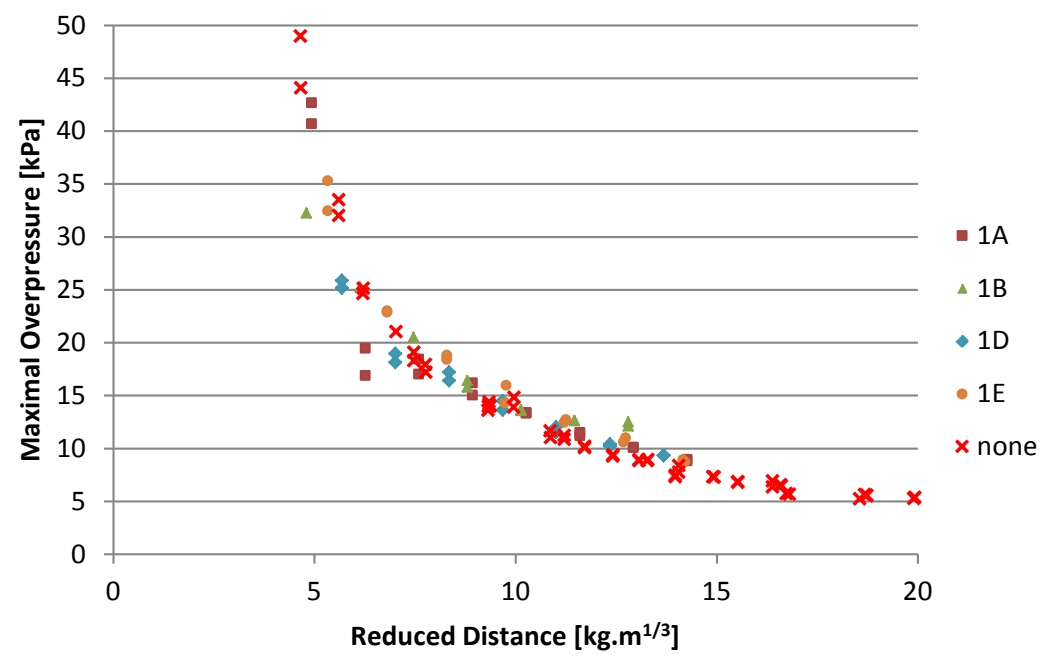

Figure 5: Comparison of measured peak overpressure with experiment without barriers (rectangular shape of barrier). 


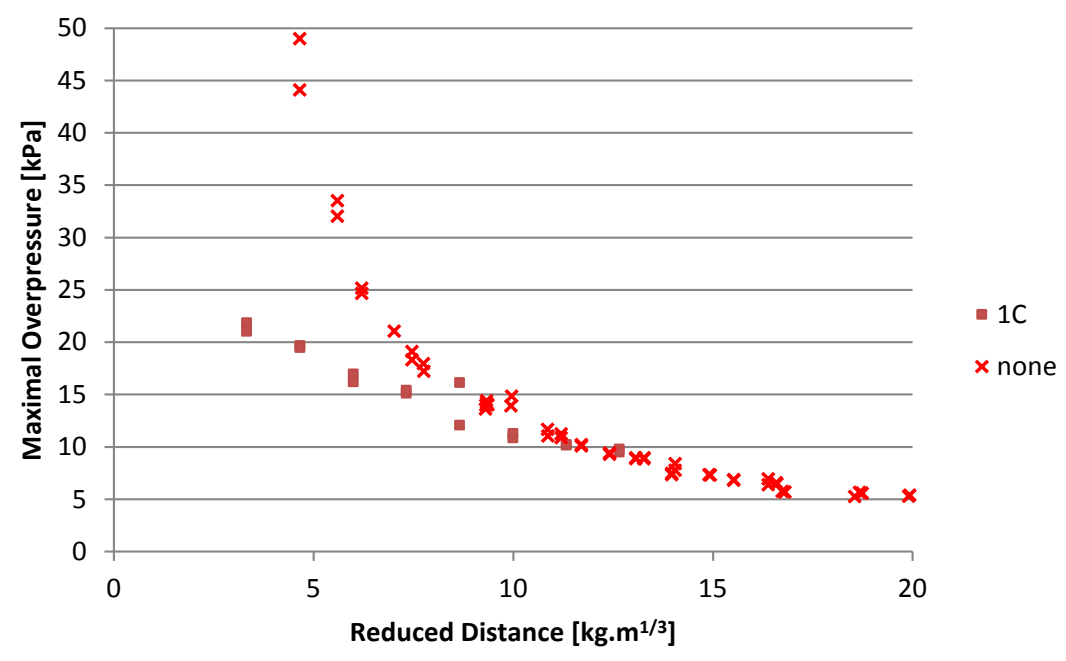

Figure 6: Comparison of measured peak overpressure with experiment without barriers (angled shape of barrier).

\section{Computer analysis}

To reduce the need for expensive experiments a computer model was developed using ANSYS and LS-DYNA softwares. The LS-DYNA solver uses explicit formulation of Finite Element Method (FEM). That means that element size and time step of the analysis must be carefully set to guarantee stable solution. The material model definitions also had to be properly calibrated to ensure accuracy of the results.

Another benefit of computer model except the price is that the solution (e.g. pressure wave propagation) is known for any position within the model unlike the experiments, in which data can only be measured in predetermined positions.

\subsection{Model calibration}

As previously mentioned pure TNT charge was used as the explosive. This means that obtained results can be easily compared with experimental data from other authors. Pachman et al. [4] conducted series of experiments with varying yield and standoff distance from the explosive charge. Foglar and Kovár [3] measured overpressure at greater distance resulting from explosion of $25 \mathrm{~kg}$ TNT charge. Both results were used for calibration of material models of air and explosive on elementary computer model without any barriers. Equations of state used to define both materials also had to be properly calibrated.

Barriers were then added and accuracy of the model with barriers was evaluated again based on data obtained from conducted experimental programme. 


\subsubsection{Meshing and element size}

A finite element mesh was created using ANSYS software. The air and explosive are modelled using ALE (Arbitrary Lagrangian-Eulerian) elements; barriers are modelled using standard solid elements.

There were two separate meshes defined; first for air and explosive, second for barriers. Hexahedral mesh is used for air and explosive, tetrahedral mesh for concrete barriers. Contact between two meshes is automatically generated by the LS-DYNA solver's function CONSTRAINED_LAGRANGE_IN_SOLID.

Mesh size and time step of the explicit dynamics solver engine have dramatic impact on model accuracy. Size of the air and explosive ALE elements was determined based on scale of the experiment and comparison of results with experimental data from Pachman et al. [4]. Satisfactory length of element edge was determined to be $25 \mathrm{~mm}$.

\subsubsection{Modelling of air and explosive materials}

Material model 009-NULL was defined for air elements and material model 008-HIGH_EXPLOSIVE_BURN was defined for the TNT charge.

Equation of state for ideal gas (3) was used to model the air. Parameters of the equation were defined according to [7].

$$
P=\left(C_{4}+C_{5} \mu\right) e_{i p v 0}=(\gamma-1) \frac{\rho}{\rho_{0}} e_{i p v 0}
$$

where: $C_{4}+C_{5}=\gamma-1=0.4$

$P_{0}=101.3 \mathrm{kPa}$

$v_{0}=1.0$

$\mathrm{e}_{\mathrm{ipv} 0}=P_{0} v_{0} /(\gamma-1)$

For TNT the JWL (Jones-Wilkins-Lee) equation of state was used (4). Calibration of this equation of state is quite difficult. The parameters were derived from $[8,9]$.

$$
p=A\left(1-\frac{\omega}{R_{1}} \frac{\rho}{\rho_{0}}\right) e^{-R_{1} \frac{\rho_{0}}{\rho}}+B\left(1-\frac{\omega}{R_{2}} \frac{\rho}{\rho_{0}}\right) e^{-R_{2} \frac{\rho_{0}}{\rho}}+\omega E \frac{\rho}{\rho_{0}}
$$

Detonation time and point of origin of the explosion is controlled by the function INITIAL_DETONATION.

\subsubsection{Modelling of concrete barriers}

Barrier deformations could have an unintended impact on results. It was decided to model the concrete barriers at this stage of research as rigid structures. Simplified material model of barrier is linear elastic, but the value of modulus of elasticity $\mathrm{E}$ is significantly increased to provide rigid-like behaviour. Interaction between air and barrier was in question, but results clearly prove that blast wave is reflected of the barrier surface properly (Figure 7). 

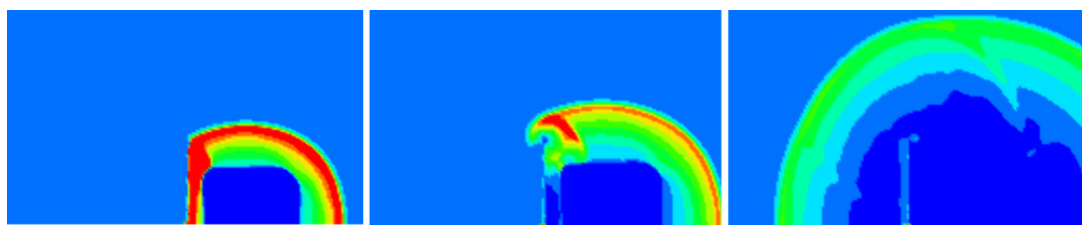

Figure 7: Contours of overpressure: Blast wave interacting with rigid barrier.

\subsection{Validation of computer models with experimental results}

During experiment multiple pressure sensors were used to measure the peak overpressure at the front of passing air blast wave. For each arrangement sensors were placed in multiple distances with step of $1000 \mathrm{~mm}$ as shown in Figure 1. Values of element pressure at corresponding coordinates were obtained from the FEM model and compared to experimental data. The comparison for some of the arrangements and standoff distances is summarized in Figure 8 and Figure 9.

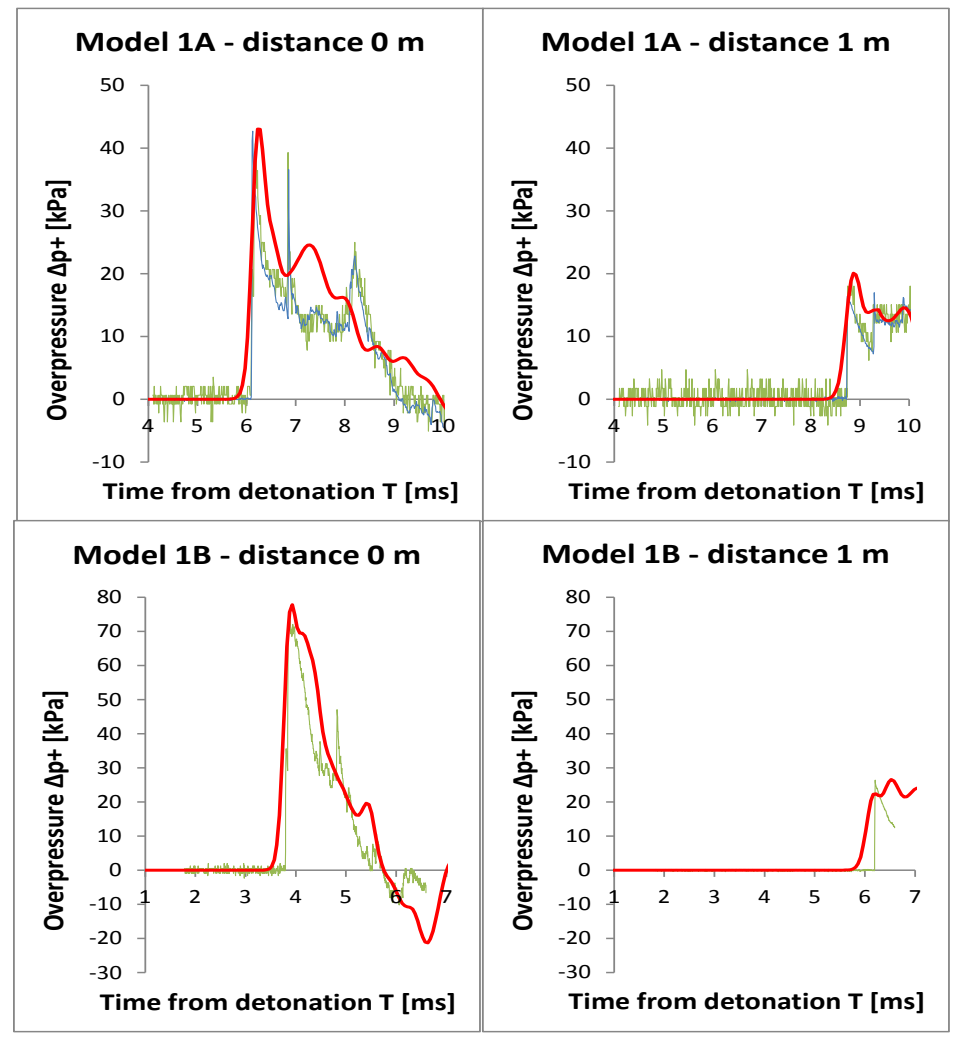

Figure 8: Comparison of measured and computed overpressure behind barrier for arrangements $1 \mathrm{~A}$ and $1 \mathrm{~B}$. (FEM results in red, experiments in blue and green). 


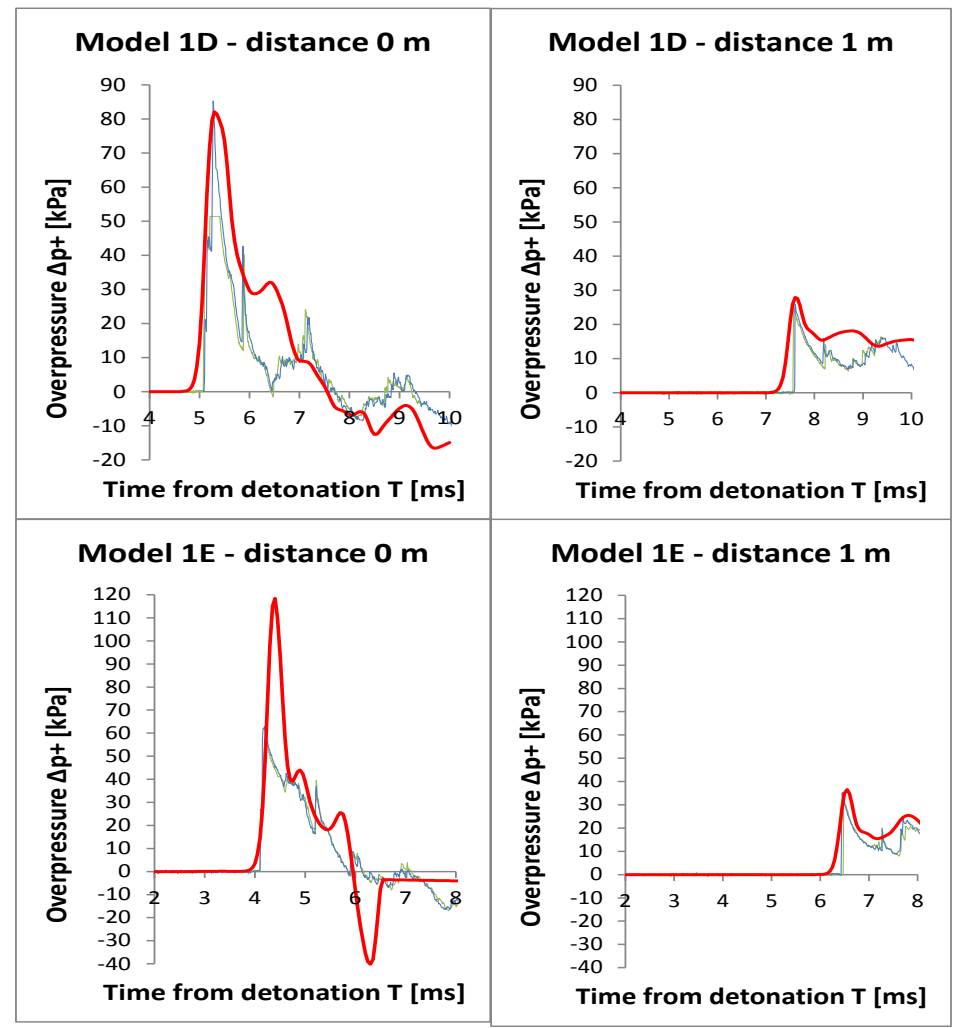

Figure 9: Comparison of measured and computed overpressure behind barrier for arrangements $1 \mathrm{D}$ and $1 \mathrm{E}$. (FEM results in red, experiments in blue and green).

Credibility of the results is strongly conditioned by proper configuration of the model. Properly calibrated model can be useful when optimizing the barrier arrangement because it can dramatically reduce cost of the experiment.

Overall the results indicate that computer model is capable of predicting the peak overpressure with relatively high accuracy in most cases. The time difference between peaks in standoff distance of $0 \mathrm{~m}$ and $1 \mathrm{~m}$ was also predicted correctly.

Based on acquired data it can be assumed that model is calibrated correctly enough to be used for optimization of barrier shape and arrangement. This way a large number of possibilities can be studied without the need for many expensive experiments.

Comparison of computer modelling results and experimental data shows that FEM modelling of blast event can in certain cases and for certain purposes substitute experimental measuring. On the other hand at least one validation test should still be performed for each change in materials or major change in arrangement of the experiment. 


\subsection{Rigid barrier effectiveness}

Although experimental results (Figure 5) show little efficiency it is clear that even rigid rectangular barriers must have some effect on pressure wave mitigation, as stated in [1]. Detailed FEM evaluation was conducted in order to determine the size of barrier "shadow" in which the overpressure is effectively reduced.
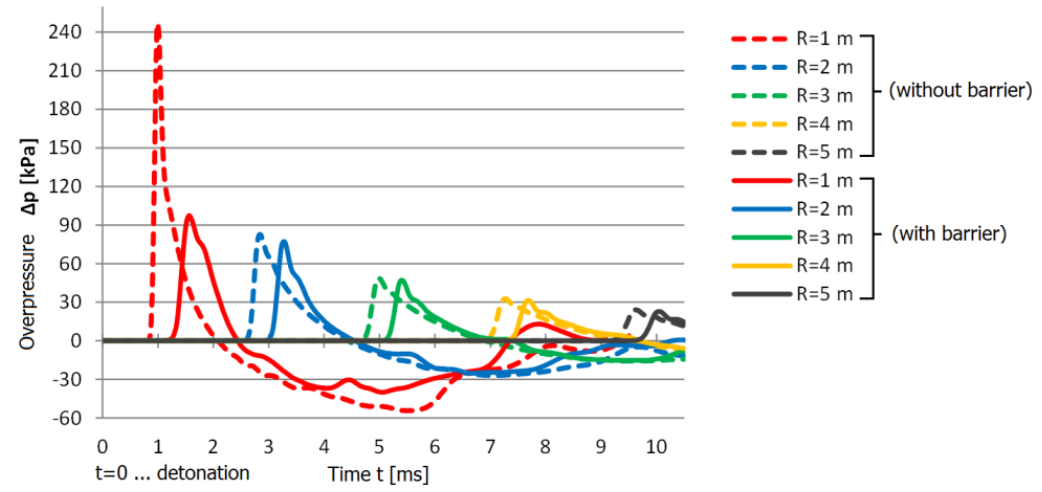

Figure 10: Comparison of shape of air shock wave behind a barrier and without barrier (in multiple distances).

A model similar to Figure 7 was created. A small TNT charge was modelled in front of the barrier. Height of the barrier is 1.2 metres. Overpressure was obtained for multiple positions. Two sets of data were obtained (with barrier and without barrier).

Results show, that efficiency of overpressure reduction is noticeable in the area directly behind the barrier. With greater distance from the barrier, the effect of overpressure reduction quickly becomes negligible (Figure 10).

Although the peak overpressure is not reduced in greater distance, the shock wave is noticeably slowed by the barriers. That too can result in reduction of the possibility of injury.

\section{Conclusions}

Although experiments were executed as planned the results failed to prove the desired high effectiveness of solid rectangular barriers. However the angled design of barrier type $1 \mathrm{C}$ showed some optimistic results and will most likely determine direction of further research in field of barrier arrangement optimization.

The results also indicate that even if the barriers have some effect on lowering the peak overpressure, that effect is limited to the area directly behind the barrier. In greater distance the effect diminishes rapidly and pressure wave resumes its original strength. That leads to the assumption, that very effective reduction of peak overpressure in greater area is not achievable with rigid barriers. The main 
benefit of such barriers would be the reduction of shock wave velocity and more importantly absorption of potentially harmful flying debris carried by the blast winds.

Comparison of computer modelling results and experimental data shows that FEM modelling of blast event can in certain cases and for certain purposes substitute experimental measuring. Credibility of the results is conditioned by proper configuration of the model. Properly calibrated model can be useful when optimizing the barrier arrangement because it can dramatically reduce cost of the experiment.

On the other hand at least one validation test should still be performed for each change in materials or major change in arrangement of the experiment. If satisfactory agreement between experiment and numerical model is achieved, the need for expensive experiments would be reduced.

\section{Acknowledgements}

The financial support of the CTU grant no. SGS13/035/OHK1/1T/11, Ministry of the Interior of the Czech Republic project no. VG20132015114 and the Czech Republic Grant Agency project no. 13-30441S is kindly acknowledged.

\section{References}

[1] Zhou, X.Q., Hao, H. Prediction of airblast loads on structures behind a protective barrier. International Journal of Impact Engineering, Volume 35, Issue 5, May 2008, pp. 363-375, ISSN 0734-743X.

[2] Wells, Geoff L. Major hazards and their management. London: Institution of Chemical Engineers. 1997, p. 305 ISBN 9780852953686.

[3] Foglar, M.; Kováŕ, M. Conclusions from experimental testing of blast resistance of FRC and RC bridge decks, International Journal of Impact Engineering, Volume 59, September 2013, pp. 18-28, ISSN 0734-743X.

[4] Pachman, J., Šelešovský, J. \& Künzel, M. (2013) Blast wave parameters of small charges: trinitrotoluene (TNT) and urea nitrate (UNi), in: Proc. of New trends in Research of Energetic Materials (NTREM), Pardubice, 10-12 April 2013, pp. 818-826.

[5] Henrych, J. (1973) Dynamika výbuchu a její užití. Academia, Prague. p. 412.

[6] Makovička, D. \& Janovský, B. (2008) Handbook of antiexplosion protection of structures, CTU Press, Prague. ISBN 978-80-01-04090-4.

[7] Huang, Y. \& Willford, M. R. (2012) Validation of LS-DYNA® MMALE with Blast Experiments. In: 12th International LS-DYNA Users Conference. Detroit, USA, 3-5 June 2012.

[8] Zukas, J. A. \& Walters, W. P. (1997) Explosive Effects and Applications. Springer-Verlag, New York. p. 460 ISBN 9780387982014.

[9] Toussaint, G. \& Durocher, R. (2008) Finite Element Simulation using SPH Particles as Loading on Typical Light Armoured Vehicles, in: Proceedings of 10th International LS-DYNA Users Conference, Dearborn, MI, USA, 8-10 June 2008. ISBN 0-9778540-4-3. 